\title{
Three Generations and Fermion Chirality from Universal Bifurcations
}

\author{
Ervin Goldfain \\ Research Scholar, Ronin Institute, Montclair, New Jersey 07043 \\ Email: ervin.goldfain@ronininstitute.org
}

\begin{abstract}
The Standard Model (SM) fails to account for either the triplication of fermion families or chiral symmetry breaking in the electroweak sector. Here we show that both phenomena arise from the approach to chaos of quantum theory near the Fermi scale.
\end{abstract}

Keywords: Standard Model; fermion generations; chiral symmetry breaking; Feigenbaum route to chaos; dissipative maps; period-doubling bifurcations

\section{Introduction}

Iterated maps of the unit interval are generic models of dynamical systems in discrete time [7-8]. The standard representation of these models is based on first order difference equations having the form

$$
x_{n+1}=f\left(x_{n}, \lambda\right)
$$

where $n=1,2, \ldots$ is the iteration index and $\lambda$ a control parameter. The dynamics expressed by (1) can be either conservative or dissipative. In the former case, the function (1) is monotonic and describes a one-to-one mapping, whereas in the latter case is non- 
monotonic and describes a two-to-one mapping. Typical examples of dissipative systems include the quadratic map and unimodal maps [1-5]. In 1978, Feigenbaum has discovered that the onset of chaos in quadratic maps occurs through period-doubling bifurcations driven by changes of the control parameter [1-2]. It was later shown that the period-doubling transition to chaos with the same universal attributes develops in many multi-dimensional dissipative nonlinear systems [3-5] In particular, unimodal maps of the form [8-9]

$$
\begin{gathered}
f_{\lambda}(x)=\lambda f(x) \\
x \in[-1,1] ; f(0)=1 ; \quad \lambda<1
\end{gathered}
$$

exhibit the following behavior: for small values of $\lambda,(2)$ has a single stable fixed point and all nearby points converge to it under multiple iterations given by (1). Ramping up $\lambda$ to a critical value $\lambda_{1}$ makes the fixed point unstable and produces a new stable pair of points of period 2. Further increasing $\lambda$ to another value $\left(\lambda_{2}\right)$ bifurcates this cycle into a cycle of period 4. The bifurcation process continues with a new sequence of cycles of period $2^{j}, j \geq 3$, eventually leading to a Cantor set structure that attracts almost all the points of the interval $[-1,1]$. On letting $\lambda$ increase beyond an endpoint value $\lambda_{N}$, with $N>>1$, stable periodic orbits surface again and split up in a similar way. In the new sequence, $\lambda$ scans another series of critical values corresponding to cycles of period $3 \cdot 2^{j}$ $, j=0,1,2, \ldots$ and so on $[8-9]$. 


\section{Chaotic behavior of quantum theory near the Fermi scale}

Quantum Mechanics contains many instances where unimodal functions of the type (2) show up. For example, a quantum wave packet initially centered at $x=0$ [10]

$$
\psi(x, 0)=\exp \left(-\frac{x^{2}}{2}\right)
$$

evolves in time according to

$$
\psi(x, t)=\exp \left(-\frac{i \omega t}{2}\right) \psi(x, 0)=\exp \left(-\frac{i \omega t}{2}\right) \exp \left(-\frac{x^{2}}{2}\right)
$$

Another example is a wavefunction localized in $x$ space as in [10]

$$
\psi(x)=\frac{\sin K\left(x-x_{0}\right)}{\pi\left(x-x_{0}\right)}, K>>1
$$

whose momentum space representation is

$$
\varphi(k)=\frac{\exp \left(-i k x_{0}\right)}{\sqrt{2 \pi}}
$$

Gauge theory demands the description of physical phenomena related to (4) or (5) to be independent of any arbitrary phase factor $\exp (-i \chi)$ added to either one of them. With reference to (4), a global gauge transformation defined by the complex parameter $\lambda_{c}=\exp (-i \chi)$ assumes the form

$$
\psi^{\prime}(x, t)=\lambda_{c} \psi(x, t)=\exp (-i \chi) \psi(x, t)
$$


Following a standard procedure, we pass from Lorentzian to Euclidean coordinates using the prescription

$$
\begin{gathered}
\chi=\omega t^{\prime}=\omega\left(-i t_{E}^{\prime}\right) \\
\chi_{E}=-\omega t_{E}^{\prime}
\end{gathered}
$$

The Euclidean control parameter in the small angle approximation may be presented as

$$
\lambda_{E}=\exp \left(-\omega t_{E}^{\prime}\right) \approx 1-\omega t_{E}^{\prime}, \quad\left|\omega t_{E}^{\prime}\right|=\varepsilon<<1
$$

In the context of the minimal fractal manifold (MFM) [11], (10) highlights the connection between the Euclidean control parameter $\lambda_{E}$ and the scale-dependent deviation from spacetime dimensionality $\varepsilon(\mu)=4-D(\mu)<<1$. One concludes from (10) that the behavior of (7) is controlled by a parameter that runs with the energy scale as in $\lambda_{E}=\lambda_{E}(\mu)$. The salient point here is that, on time scales commensurate or shorter than the inverse of the electroweak scale $\left(t^{\prime} \leq O\left(M_{E W}^{-1}\right)\right)$, (8) and (10) undergo fast fluctuations inherent in the uncertainty principle.

Taken together, all these observations indicate that (7) undergoes period-doubling transition to chaos upon letting $\lambda_{E}$ scan a continuous range of values. In particular, as shown in [6],

1) Gauge bosons develop from the $2^{j}$ bifurcation pattern, while fermions from the $3 \cdot 2^{j}$ pattern. The first stage of the fermion sector $(j=0)$ contains a triplication of fermion generations, in full agreement with the experimental basis of the SM. 
2) The neutrino-antineutrino branch developed at $j=0$ displays an intrinsic chiral asymmetry upon applying the CPT operator, which entails that only one of the Left (L) and Right (R) neutrino states exist. It follows that chiral symmetry breaking is rooted in the neutrino sector and stems from the transition to chaos driven by the global gauge transformation (7).

Both findings are consistent with [11-12], where the SM is conjectured to represent a selfcontained multifractal set, whose composition is constrained by the so-called "sum-ofsquares" relationship. The violation of chiral symmetry falls in line with spacetime anisotropy induced by fractional differential and integral operators near or above the Fermi scale [13].

In the same context, we point out that the sequential generation of SM families may be interpreted from a different vantage point. As discussed in [20], the quartet of electroweak bosons bifurcates into the gluon octet and the lepton multiplet into the quark multiplet according to

$$
\begin{gathered}
\left(\begin{array}{llll}
\gamma & W^{-} & W^{+} & Z^{0}
\end{array}\right) \Rightarrow\left(\begin{array}{llll}
\text { gluons }_{1-8}
\end{array}\right) \\
\left(\begin{array}{llllllll}
v_{e} & v_{\mu} & v_{\tau} & e & \mu & \tau
\end{array}\right)+\text { antiparticles } \Rightarrow\left(\begin{array}{llllll}
u & d & c & s & b & t
\end{array}\right)_{r, g}+\text { antiparticles }
\end{gathered}
$$

In particular, (11) shows that the dynamical transition $U(1) \times S U(2) \Rightarrow S U(3)$ is a transformation of a stable cycle of period 4 in the electroweak sector to a stable cycle of period 8 in the strong sector. It is apparent that this viewpoint complies with the gauge group structure of the SM. 
In closing, we note that the wide window separating the gauge boson and fermion bifurcation patterns may be likely attributed to the Cantor Dust composition of Dark Matter [14-19]

\section{$\underline{\text { References }}$}

1. M. J. Feigenbaum, "Quantitative universality for a class of nonlinear transformations", J. Stat. Phys. 19:25-52 (1978).

2. M. J. Feigenbaum, "The universal metric properties of nonlinear transformations”, J. Stat. Phys. 21:669-706 (1979).

3. P. Cvitanovic (ed.), "Universality in Chaos", 2nd Ed. (Adam Bilger, Boston, 1989).

4. P. Collet, J.-P. Eckmann, and H. Koch, "Period Doubling Bifurcations for Families of Maps on Rn”, J. Stat. Phys. 25:1-14 (1981)

5. http://spkuz.narod.ru/2005JSP.pdf

6. E. Goldfain, "A Bifurcation Model of the Quantum Field", Physica A 165: 399-419 (1990). A copy of this reference is available at: https://www.academia.edu/38767540/A bifurcation model of the quantum field

7. P.Collet, J.-P. Eckman, "Iterated Maps on the Interval as Dynamical Systems", (Birkhäuser, Boston, 1980)

8. E. A. Jackson, "Perspectives of nonlinear dynamics", (Cambridge Univ. Press, 1991).

9. K. J. Falconer, “The geometry of fractal sets”, (Cambridge Univ. Press, 1988).

10. D. Bohm, "Quantum Theory”, (Dover, New York, 1989).

11. Available at the following sites: 
http://www.aracneeditrice.it/aracneweb/index.php/pubblicazione.html?item=9788854 $\underline{889972}$

https://www.researchgate.net/publication/278849474 Introduction to Fractional Fi eld Theory consolidated version

12. https://www.researchgate.net/publication/343426122 Derivation of the Sum-of$\underline{\text { Squares Relationship }}$

13. https://www.sciencedirect.com/science/article/abs/pii/S1007570406001183

14. Available at the following site:

https://www.researchgate.net/publication/300402085 Fractional Field Theory and Physics of the Dark Sector

15. Available at the following site:

https://www.researchgate.net/publication/343426110 Fractional Spacetime the Em ergence_of the_Dark_Sector_II\#fullTextFileContent

16. Available at the following site:

https://www.researchgate.net/publication/343425902_Fractional Spacetime the Em ergence of the_Dark_Sector_I

17. Available at the following site:

https://www.researchgate.net/publication/343426172 Anyon Physics and the Topo logy of Dark Matter

18. Available at the following site:

https://www.researchgate.net/publication/343426202 Minimal Fractal Manifold an

d the Dual Nature of Dark Matter

19. Available at the following site: 
https://www.researchgate.net/publication/336287017 Cantor Dust as Underlying Texture of Fuzzy_Dark_Matter

20. Available at the following site:

https://www.researchgate.net/publication/343403707 Emergence of Lie Groups G auge Symmetries from Complex Dynamics 